Published in final edited form as:

Ann Intern Med. 2016 October 04; 165(7): 523-524. doi:10.7326/M16-1581.

\title{
Predicting the Prognosis of Acute Pancreatitis
}

\author{
Chris E. Forsmark, MD and \\ University of Florida Gainesville, Florida \\ Dhiraj Yadav, MD, MPH \\ University of Pittsburgh School of Medicine, Pittsburgh, Pennsylvania
}

\begin{abstract}
Clinicians find value in predicting the prognosis and course of disease in individual patients. All diseases have intrinsic variability in risk, severity, and outcome, which is driven by differences in individual susceptibility, general health, and physiologic reserve, as well as in the processes and pathogenicity of the disease. For example, patients with gastrointestinal bleeding due to peptic ulcer may be stratified into high- and low-risk groups through the use of scoring systems to assess patient characteristics and bleeding volume, and by the ulcer's appearance during initial endoscopy. This stratification allows clinicians to triage patients, identify the appropriate therapy, and determine the duration of (and even need for) hospitalization (1).
\end{abstract}

Acute pancreatitis (AP) is increasing in incidence (2) and is among the most common reasons for inpatient hospitalization for a gastrointestinal condition in the United States (3). The outcome of this disease is quite variable: approximately $80 \%$ of patients have a shortlived, mild illness and recover promptly, whereas $20 \%$ have a more serious illness characterized by organ system failure, pancreatic necrosis, various infections, and death (4). The overall mortality of AP is now less than $2 \%$ in the United States; however, among those with severe disease, the rate may approach $20 \%$ to $30 \%$ (4). Therefore, predicting which patients will have more severe disease, as well as who might die, may be helpful in the triage of patients to high-intensity nursing units and in determining whether more aggressive therapy should be applied early in the clinical course.

There has been a long-standing interest in developing tools to assist clinicians in predicting severe AP before it develops. These instruments include specific physical findings, laboratory tests, cross-sectional imaging, and various multiple-factor scoring systems. Within each scoring system, different cutoff scores may be chosen to differentiate high-risk from low-risk patients. These systems have been hampered in that they often identify severe disease only as it develops, do not predict severe disease with enough lead time for potential intervention, or are too complex for everyday use. Although any medical student is familiar

\footnotetext{
Requests for Single Reprints: Chris E. Forsmark, MD, Division of Gastroenterology, Hepatology, and Nutrition, University of Florida, Box 100214, 1329 Southwest 16th Street, Suite 5251, Gainesville, FL 32610; chris.forsmark@medicine.ufl.edu.

Current Author Addresses: Dr. Forsmark: Division of Gastroenterology, Hepatology, and Nutrition, University of Florida, Box 100214, 1329 Southwest 16th Street, Suite 5251, Gainesville, FL 32610.

Dr. Yadav: Division of Gastroenterology, Hepatology and Nutrition, University of Pittsburgh School of Medicine, 200 Lothrop Street, M-2, C-Wing, Pittsburgh, PA 15213.
}

Disclosures: Dr. Yadav has served as a consultant for AbbVie and has received royalties from Up-To-Date, outside the submitted work. Disclosures can be viewed at www.acponline.org/authors/icmje/ConflictOfInterestForms.do?msNum=M16-1581. 
with the Ranson criteria, most of us have yet to meet a trainee who can remember or recite the 11 measures.

Di and colleagues (5) carefully performed a systematic review of the 18 multiple-factor scoring systems proposed to date and found them to be of limited accuracy or clinical value in predicting mortality. Similarly, the incremental benefit from using more than 1 of these systems, evaluated in only a few of the studies reviewed, also is limited. Among the reasons the authors identified for these conclusions, the most important are poor quality of the studies (see the authors' Table 5 in Supplement 1, which is available at www.annals.org), heterogeneity in patient populations, the clinical setting and influence of treatment administered, and a lack of information on transfer status (whether the patient was directly admitted or transferred from a referring hospital). Although many of these limitations may be overcome by a head-to-head comparison of scoring systems in the same patient population, 1 such study found the existing systems to be only modestly accurate in predicting persistent organ failure, and concluded that they may have reached their maximal efficacy (6). Of interest, several simple, routinely available clinical measures, such as serum hematocrit, elevations in blood urea nitrogen, and presence of systemic inflammatory response syndrome (especially lasting more than 48 hours) $(6,7)$, may be as accurate as the more complex multiple-factor scoring systems in predicting severe AP.

Advances in the understanding of AP pathophysiology and natural history have revealed 2 disease phases (early [in the first 7 days] and late [after 7 days]) and 2 primary determinants of mortality and clinically relevant outcomes (persistent organ failure and pancreatic necrosis on contrast-enhanced computed tomography), which are linked to each other. Persistent organ failure (that is, organ failure lasting more than 48 hours) is often associated with pancreatic necrosis, but it may occur solely from a systemic inflammatory response to pancreatic injury. It is the primary cause of death in the early phase. Pancreatic necrosis often drives organ failure, and infected pancreatic necrosis (typically occurring after 2 weeks) and hospital-associated infections are the main causes of late mortality (8). The revised Atlanta classification system (9), a recently published international consensus document, stratifies AP into 3 severity categories: severe (presence of persistent organ failure-pulmonary, renal, cardiovascular, or multi-system — as measured by a modified Marshall scoring system), moderate (transient organ failure, or local or systemic complications without persistent organ failure), and mild (no organ failure and no local or systemic complications). Patients with severe disease account for almost all deaths due to AP. Those with moderate or severe disease account for almost all morbidity, particularly from local complications, such as pancreatic necrosis. The revised Atlanta criteria also define the various local complications more precisely, and interested readers are referred to those definitions. A separate consensus group generally agreed with the Atlanta system (10) but also noted that the presence of both persistent organ system failure and infected necrosis, which the group termed "critical" pancreatitis, leads to greater mortality than either condition alone. These efforts provide much clearer and more quantifiable definitions of severe AP.

For clinicians, knowledge of the primary role of persistent organ failure and supporting role of pancreatic necrosis in determining AP outcomes is important. These outcomes include not 
only death, but also non-fatal illness that prolongs hospital stays and increases health care costs. Although the new Atlanta classification system does not provide methods to predict disease severity (9), it simplifies decision making for busy physicians while providing a clear idea of the expected course in a given patient. The best predictor of severe disease is not one of the multiple-factor systems, but rather an experienced clinician who is informed by patient- and disease-related factors, such as older age, obesity (particularly morbid obesity), comorbidities, and alcohol use, and cognizant of the predictive value of the simple measures mentioned earlier (namely, systemic inflammatory response syndrome and blood urea nitrogen, creatinine, and hematocrit levels). All of these factors are associated with worse outcomes and help in making decisions regarding patient triage and ongoing care.

Regarding research, we believe that developing new multiple-factor clinical scoring systems will not increase accuracy in prediction, and that all current systems are inadequate to guide clinical judgment. Future studies should focus on the translational research needed to identify better biological markers of the primary determinants of outcome-that is, organ failure and pancreatic necrosis - as well as host-related factors that influence the severity of the systemic response to pancreatic necrosis. Such an approach might help in understanding the mechanisms of severe disease, identifying at-risk patients soon after or before pancreatic injury occurs, and developing therapies to mitigate disease severity.

\section{References}

1. Rockall TA, Logan RF, Devlin HB, Northfield TC. Selection of patients for early discharge or outpatient care after acute upper gastrointestinal haemorrhage. National Audit of Acute Upper Gastrointestinal Haemorrhage. Lancet. 1996; 347:1138-40. [PubMed: 8609747]

2. Yadav D, Lowenfels AB. Trends in the epidemiology of the first attack of acute pancreatitis: a systematic review. Pancreas. 2006; 33:323-30. [PubMed: 17079934]

3. Peery AF, Crockett SD, Barritt AS, Dellon ES, Eluri S, Gangarosa LM, et al. Burden of gastrointestinal, liver, and pancreatic diseases in the United States. Gastroenterology. 2015; 149:1731-1741 e3. DOI: 10.1053/j.gastro.2015.08.045 [PubMed: 26327134]

4. Banks PA, Freeman ML. Practice Parameters Committee of the American College of Gastroenterology. Practice guidelines in acute pancreatitis. Am J Gastroenterol. 2006; 101:2379_ 400. [PubMed: 17032204]

5. Di MY, Liu H, Yang ZY, Bonis PAL, Tang JL, Lau J. Prediction models of mortality in acute pancreatitis in adults: a systematic review. Ann Intern Med. 2016; Epub ahead of print. doi: 10.7326/M16-0650

6. Mounzer R, Langmead CJ, Wu BU, Evans AC, Bishehsari F, Muddana V, et al. Comparison of existing clinical scoring systems to predict persistent organ failure in patients with acute pancreatitis. Gastroenterology. 2012; 142:1476-82. quiz e15-6. DOI: 10.1053/j.gastro.2012.03.005 [PubMed: 22425589]

7. Koutroumpakis E, Wu BU, Bakker OJ, Dudekula A, Singh VK, Besselink MG, et al. Admission hematocrit and rise in blood urea nitrogen at $24 \mathrm{~h}$ outperform other laboratory markers in predicting persistent organ failure and pancreatic necrosis in acute pancreatitis: a post hoc analysis of three large prospective databases. Am J Gastroenterol. 2015; 110:1707-16. DOI: 10.1038/ajg.2015.370 [PubMed: 26553208]

8. Renner IG, Savage WT 3rd, Pantoja JL, Renner VJ. Death due to acute pancreatitis. A retrospective analysis of 405 autopsy cases. Dig Dis Sci. 1985; 30:1005-18. [PubMed: 3896700]

9. Banks PA, Bollen TL, Dervenis C, Gooszen HG, Johnson CD, Sarr MG, et al. Acute Pancreatitis Classification Working Group. Classification of acute pancreatitis-2012: revision of the Atlanta classification and definitions by international consensus. Gut. 2013; 62:102-11. DOI: 10.1136/ gutjnl-2012-302779 [PubMed: 23100216] 
10. Dellinger EP, Forsmark CE, Layer P, Lévy P, Maraví-Poma E, Petrov MS, et al. Pancreatitis Across Nations Clinical Research and Education Alliance (PANCREA). Determinant-based classification of acute pancreatitis severity: an international multidisciplinary consultation. Ann Surg. 2012;

256:875-80. DOI: 10.1097/SLA.0b013e318256f778 [PubMed: 22735715] 